\title{
Investigation of Resonance Structure in the System of two KS Mesons in the Mass Regions around 2230 $\mathrm{MeV}$
}

\author{
Ekaterina Fadeeva*广 \\ ITEP, Russia \\ E-mail: fadeeva@itep.ru
}

\begin{abstract}
This report is devoted to narrow resonance in the $K_{S} K_{S}$-system that is observed in experimental data coming from 6-m spectrometer (MIS ITEP). The experimental data on the production of $K_{S}$ pair were obtained in $\pi^{-} p$ interaction at $40 \mathrm{GeV}$ by using a neutral trigger. In the $K_{S} K_{S^{-}}$-system a maximum of width $<8 \mathrm{MeV}$ is observed at mass of about $2230 \mathrm{MeV}$. The statistical confidence of this state is better than 6 standard deviations. Spin-parity of the state $\mathrm{X}(2230)$ is preferably $J^{P C}=2^{++}$. Seeing its very small width this resonance is likely to be cryptoexotics (see [1],[2] for details)
\end{abstract}

International Europhysics Conference on High Energy Physics

July 21st - 27th 2005

Lisboa, Portugal

*Speaker.

${ }^{\dagger}$ I.A.Erofeev, O.N.Erofeeva, V.K.Grigor’ev, Yu.V.Katinov, V.I.Lisin, V.N.Luzin, V.N.Nozdrachev, Yu.P.Shkurenko, V.V.Sokolovsky, V.V.Vladimirsky. ITEP, Moscow. 


\section{Introduction}

The experimental data we analyze had been accumulated by using 6-m spectrometer developed at the Institute for Theoretical and Experimental Physics (ITEP, Moscow) and installed at a $40 \mathrm{GeV}$ $\pi^{-}$-meson beam from the accelerator at the Institute for High Energy Physics (IHEP, Serpukhov). A detailed description of the 6-m spectrometer is presented in [3]. The system of two $K_{S}$-mesons that was recorded under the experimental conditions of the $6-\mathrm{m}$ spectrometer is produced in the following two reactions:

$$
\begin{gathered}
\pi^{-} p \rightarrow K_{S} K_{S} n \\
\pi^{-} p \rightarrow K_{S} K_{S}+\left(n+m \pi^{0}, p+\pi^{-}, \ldots\right) .
\end{gathered}
$$

Reaction 1.1 is separated with a trigger facility based on veto counters surrounding the liquidhydrogen target. Due to imperfect trigger operation, some fraction of events of the reaction 1.2 is recorded by the setup. The spectrometer records with high efficiency $K_{S}$-mesons going to the forward direction and decaying into to charged $\pi$-mesons. The precision of the measurement of the effective mass of the $K_{S} K_{S}$-system is better than $8 \mathrm{MeV}$ in mass region around $2200 \mathrm{MeV}$. The recording efficiency is about $40 \%$ for the $K_{S} K_{S^{-}}$-system in the mass region around $2200 \mathrm{MeV}$. It depends on the $K_{S}$-meson momenta.

In the analysis of the $K_{S} K_{S}$-system we used the following kinematical variables: the effective mass $M_{K K}$ of the pair of $K_{S}$-mesons; the missing mass squared $M M^{2}$ defined as the squared mass of particles that are produced together with the $K_{S} K_{S}$-system and which are not recorded in the spectrometer; the 4-momentum transferred from the beam to the system being studied, $t$; the cosine of the Gottfried-Jackson angle, $\cos \theta_{G J}$; the Treiman-Yang angle, $\phi_{T Y}$.

The angles are calculated in the rest frame of the pair of $K_{S}$-mesons, the beam axis direction in this system being taken for the polar axis. The plane from which the Treiman-Yang angle is reckoned is spanned by the momenta of the beam and of the target proton in this reference frame.

\section{Resonance $X(2230)$}

The Figure 1 shows the mass spectrum of the $K_{S} K_{S}$-system from 2100 to $2450 \mathrm{MeV}$ and from 2170 to $2280 \mathrm{MeV}$ with the bin width being 20 and $5 \mathrm{MeV}$, respectively. The resonance feature manifests itself as a maximum in the vicinity of $2230 \mathrm{MeV}$.

The figure 2 represents the distribution of number of events with respect to the transferred momentum. Momentum-transfer distribution of events from the effective mass region of the $K_{S} K_{S}$-system that are adjacent to the resonance region from the left and from the right $\left(2170<M_{K K}<2210,2236<\right.$

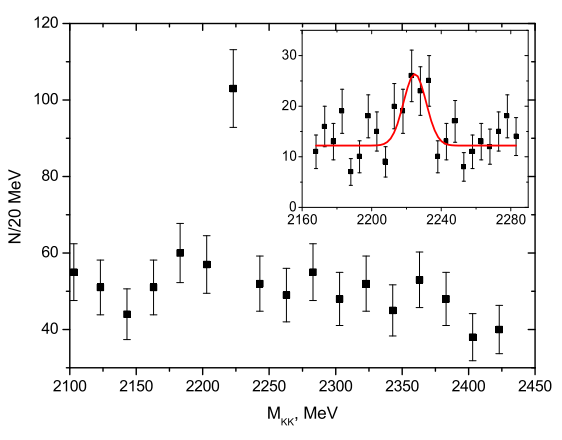

Figure 1: Effective-mass spectrum of two $K_{S}$-mesons. 
curve and dots and of events from the resonance region $\left(2213<M_{K K}<2233(\mathrm{MeV})\right)$ only by the dots. It is seen that these distributions are quite different.

In order to determine the parameters of the observed resonance feature and its statistical confidence, the experimental data were fitted by the Maximum-likelihood method (MLM). The main advantage of this method over the histogramming is that the mass and angles are not averaged over the bin width in the fitting procedure, so that the result does not depend on the choice of the reference point and the number of bins into which the mass range under study is divided. Describing the experimental data, we used the probabilitydensity function $F(P ; \Omega)$, where $P$ is the

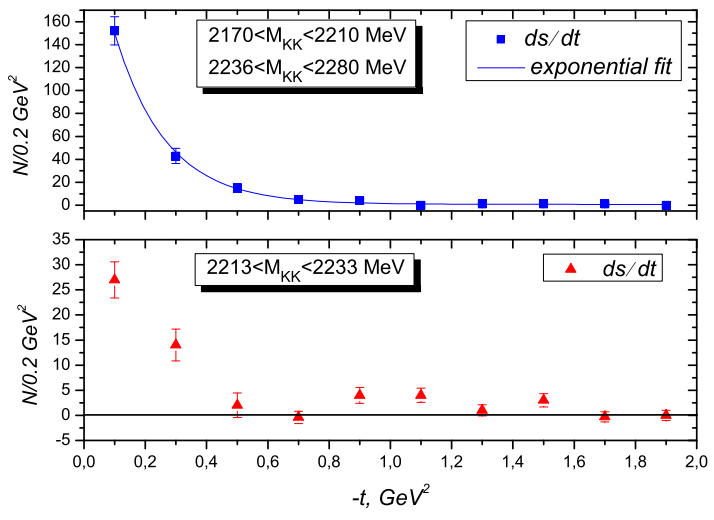

Figure 2: Momentum-transfer distribution for X(2230). set of the parameters (the amplitude, the mass $M$, the width $\sigma$ appearing in the Gauss function and the coefficients of the squared amplitudes of the angular distributions). Elements of the phase space $\Omega$ are effective mass of two $K_{S}$-mesons, the cosine of the Gottfried-Jackson angle $\cos \theta_{G J}$, the Treiman-Yang angle $\phi_{T Y}$.

Fitting was performed for events falling within the range $2100-2350 \mathrm{MeV}$ of $K_{S} K_{S}$ masses. There were 711 events in this range. A first-degree polynomial proved to be sufficient for describing the mass dependence of the background. The resonance was approximated by a Gauss function. In order to obtain the most probable values of the parameters we minimized the functional:

$$
\int_{\Omega} \varepsilon(\Omega) F(P ; \Omega) d \Omega-\sum_{i=1}^{N} \ln F\left(P ; \Omega_{i}\right) .
$$

where $\varepsilon(\Omega)$ is the event-detection recording, $N$ being the number of events. To compare the probabilities of experimental-data description with different parameter set, we calculated $\chi^{2}$ by the formula:

$$
\chi^{2}=-2 \ln L+\text { const }
$$

where $L=\prod_{i=1}^{N} F\left(P ; \Omega_{i}\right)$. The constant was chosen in such a way that $\chi^{2}$ obtained via minimization without allowing for the Gauss function was equal to 100 . The results of various version of fitting are given in the table, where the number of events in each of the waves of background and the resonance is displayed, along with the central values of the resonance's mass and width. The last column shows the $\chi^{2}$ values, with the number of degrees of freedom $N_{d . f}$. being subtracted.

The first line of the table 1 represents the result of fitting without allowing for the Gauss function and without discarding events falling within the resonance region. In the second line is given the result of fitting in terms of the $D_{0}$ - and $D_{+}$-waves. 


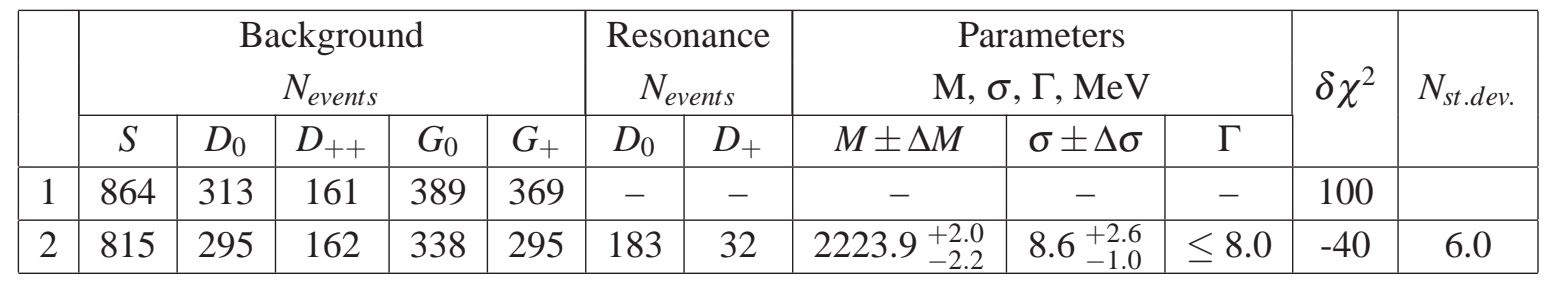

Table 1: Different sets of minimization for X(2230).

\section{Comparison with other results}

Here is presented comparison with other results

\begin{tabular}{|c|c|c|c|}
\hline TECN & Reaction & Mass, MeV & Width, MeV \\
\hline $96 \mathrm{BES}$ & $e^{+} e^{-} \rightarrow J / \psi \rightarrow \gamma \pi^{+} \pi^{-}$ & $2235^{-} \pm 4$ & $19_{-11}^{+13} \pm 12$ \\
\hline $96 \mathrm{BES}$ & $e^{+} e^{-} \rightarrow J / \psi \rightarrow \gamma K^{+} K^{-}$ & $2230_{-7}^{+6} \pm 16$ & $20_{-15}^{+20} \pm 17$ \\
\hline $96 \mathrm{BES}$ & $e^{+} e^{-} \rightarrow J / \psi \rightarrow \gamma K_{S}^{0} K_{S}^{0}$ & $2232_{-7}^{+8} \pm 15$ & $20_{-16}^{+25} \pm 14$ \\
\hline $96 \mathrm{BES}$ & $e^{+} e^{-} \rightarrow J / \psi \rightarrow \gamma p \bar{p}$ & $2235^{-} \pm 4 \pm 5$ & $15_{-9}^{+12} \pm 9$ \\
\hline $88 \mathrm{LASS}$ & $11 K^{-} p \rightarrow K^{+} K^{-} \Lambda$ & $2209_{-15}^{+17} \pm 10$ & $6_{-57}^{+107}$ \\
\hline $88 \mathrm{SPEC}$ & $40 \pi^{-} p \rightarrow K_{S} K_{S} n$ & $2230 \pm 20$ & $80 \pm 30$ \\
\hline $86 \mathrm{MRK} 3$ & $e^{+} e^{-} \rightarrow \gamma K^{+} K^{-}$ & $2230 \pm 6 \pm 14$ & $26_{-16}^{+20} \pm 17$ \\
\hline $86 \mathrm{MRK} 3$ & $e^{+} e^{-} \rightarrow \gamma K_{S}^{0} K_{S}^{0}$ & $2232^{-1} \pm 7 \pm 7$ & $18_{-15}^{+23} \pm 170$ \\
\hline $05 \mathrm{SPEC}$ & $40 \pi^{-} p \rightarrow K_{S} K_{S} n$ & $2223.9_{-2.2}^{+2.0} \pm 5$ & $\leq 8$ \\
\hline
\end{tabular}

\section{Conclusions}

Let us summarize results. At the confidence better than 6 standard deviation we have obtained an indication of the existence of the resonance feature having a mass $2223.9_{-2.2}^{+2.0} \pm 5.0 \mathrm{MeV}$ and a width less than $8 \mathrm{MeV}$. The spin-parity of this resonance is preferably $J^{P C}=2^{++}$The product of the cross section for $\mathrm{X}(2230)$ formation and the relevant branching ratio $\sigma \dot{B} R\left(K_{S} K_{S}\right)$ is estimated at about $9 \pm 5 \mathrm{nbn}$

Main feature of this resonance is its very small width $(<8 \mathrm{MeV})$ and extraordinary cross-section dependence on the transferred momentum.

\section{References}

[1] Landsberg L.G., Yad. Phys. 57, 47 (1994); Phys. Atom. Nucl. 57, 42 (1994).

[2] Landsberg L.G., Usp. Phys. Nauk 164, 1129 (1994); Phys. Uspekhi 37, 1043 (1994).

[3] Nozdrachev V.N. et al., "The resonance structures of $K_{S} K_{S}$ and $\Lambda \bar{\Lambda}$ spectrum at MIS ITEP", in CP619, Hadron Spectroscopy: Ninth International Conference, edited by D. Amelin and A.M. Zaitsev, 2001, pp. 155-164. 